\section{Avaliação antropométrica de pré-escolares do município de Mogi-Guaçú, São Paulo: subsídio para políticas públicas de saúde}

\section{Anthropometric assessment in preschool children in Mogi-Guaçu, State of São Paulo: a support for public health policies}

Isidoro Tadeu Fernandes 1

Paulo Rogério Gallo 2

Alberto Olavo Advíncula 3

\section{Abstract}

Objectives: to assess anthropometrically préschool children nutritional status from the Municipal Child Education School system (EMEI's) in MogiGuaçu, São Paulo, Brazil.

Methods: based on a per conglomerate sample, estimated by equiprobability with 347 children from three to seven years old enrolled in nine of the 38 EMEI's in the survey. Individual measurements of weight, stature, complemented by social and economical data questionnaire. Measurements were controlled with the use of the $z$ score and compared with the referential established by the National Health Statistical Center of the United States of America, and body mass index (BMI).

Results: a probable median dislocation of anthropometric indexes to the right of the normal curve related to the $z$ score of weight for age indicators (+0.54; sd 1.48), height for age (+0.20; sd 1.02), BMI (+0.30; sd 1.13).

Conclusions: the nutritional status of the children enrolled in the EMEI's does not point toward energy protein malnutrition (EPM). On the other hand, a problem of overweight and obesity was determined in the focused age group.

Key words Growth, Child development, Nutritional status, Body mass index, Anthropometry

\section{Resumo}

Objetivos: analisar o estado nutricional, através de avaliação antropométrica, dos pré-escolares que freqüentam Escolas Municipais de Educação Infantil (EMEIs) de Mogi-Guaçú, São Paulo, Brasil.

Métodos: a partir de uma amostra por conglomerado, estimada pela equiprobabilidade, com participação de 347 crianças de três a sete anos de idade, matriculadas em nove EMEIs, das 38 existentes na pesquisa, foram tomadas medidas individuais de peso e estatura, complementadas por questionário com informações socioeconômicas familiares. As medidas foram controladas com o uso de escores z e comparadas com o referencial do Centro Nacional de Estatísticas da Saúde dos Estados Unidos da América, e o índice de massa corporal.

Resultados: verificou-se um provável deslocamento da média dos índices antropométricos para a direita da curva normal no tocante ao escore $z$ dos indicadores peso/idade $(+0,54 ; d p 1,48)$, altura/idade $(+0,20 ; d p$ 1,02), IMC (+0,30; $d p$ 1,13).

Conclusões: a caracterização da situação nutricional das crianças matriculadas nas EMEIs não aponta para risco de desnutrição protéico-energética (DPE). Em contrapartida, observou-se, como problema, a presença de sobrepeso e obesidade.

Palavras-chave Crescimento, Desenvolvimento infantil, Estado nutricional, Índice de massa corporal, Antropometria 


\section{Introdução}

O acompanhamento da situação nutricional das crianças de um país ou região constitui um instrumento essencial para a aferição das condições de saúde da população infantil. ${ }^{1}$ As medidas antropométricas são utilizadas desde o século XVIII como instrumento de avaliação da saúde, ${ }^{2}$ porém só há menos de 40 anos foram normatizadas para avaliação do estado nutricional individual e de populações. ${ }^{3}$ Neste sentido, as medidas de peso e estatura são consideradas de alta sensibilidade, particularmente durante a idade pré-escolar, para refletir variações nas condições nutricionais 4 e, indiretamente, as influências do ambiente socioeconômico.5,6 Em assim sendo, os índices antropométricos podem ser tomados como indicadores positivos de saúde, pois permitem avaliar o potencial de desenvolvimento físico alcançado. Fato interessante, que se tem observado com a utilização desse instrumento no decorrer dos últimos anos, é a inversão nas taxas de desnutrição e obesidade. Um aumento crescente na prevalência de sobrepeso e obesidade tem sido observado em adultos ${ }^{7}$ e em crianças ${ }^{8}$ em todo o mundo. Monteiro e Conde 9 confirmam no âmbito do Município de São Paulo a tendência de diminuição da desnutrição em todas as classes sociais. Esses mesmos autores, 10 estudando o crescimento linear de crianças até cinco anos de idade, mostraram que as crianças teriam crescido cerca de $3,3 \mathrm{~cm}$ em estatura na idade de 30 meses, o correspondente a 0,96 escore $z$ da relação altura/idade, em cerca de 22 anos de intervalo. Quanto às justificativas para o crescimento, os referidos autores apresentam como fatores determinantes o melhor acesso das crianças e seus familiares aos serviços de saúde, ao saneamento básico e melhoria nas condições de moradia, além de melhoria no nível educacional dos pais. ${ }^{11}$

Um estudo realizado com os dados obtidos em 1999 e 2000 como parte do IV National Health and Nutrition Examination Survey (NHANES IV) sobre prevalência de sobrepeso em crianças americanas, através da análise do índice de massa corporal (IMC) por sexo e idade, mostrou uma prevalência de sobrepeso de $10,4 \%$ entre dois e cinco anos de idade e de $15,3 \%$ entre 6 e 11 anos, havendo aumento em relação à pesquisa anterior, III NHANES. 12

O presente estudo se propõe a analisar o perfil nutricional de uma população de pré-escolares, de um município próximo à cidade de São Paulo, em que as condições de saneamento, moradia e acesso aos Serviços de Saúde estariam eqüalizadas pelas políticas públicas. 13

\section{Métodos}

Foi realizada amostragem probabilística das crianças matriculadas em nove Escolas Municipais de Educação Infantil (EMEIs) sorteadas dentre as 38 existentes na cidade de Mogi-Guaçú, São Paulo, Brasil, no momento da pesquisa. O tamanho da amostra foi calculado a partir do universo de 4068 pré-escolares matriculados e de uma proporção esperada de desnutrição entre $3,9 \%$, com pior resultado de $6,0 \%$, aceitando-se um erro alfa de $5 \%$ e poder do teste de $80 \%$. Às 309 crianças calculadas, dessa maneira, pelo programa Epi-info 6.04, acrescentou-se uma estimativa de $12 \%$ de perdas, prevendo-se portanto, uma busca de 351 pré-escolares. Ao final, o estudo contou com a participação de 347 crianças entre três e sete anos de idade. As escolas foram escolhidas por sorteio aleatório, obedecendo-se as condições de eqüiprobabilidade do número de alunos matriculados em cada escola. As medidas de peso e estatura obtidas de cada criança, de acordo com protocolo internacional, 14 e complementadas com informações coletadas em nível familiar, com dados socioeconômicos, foram armazenadas em banco de dados Excel. As medidas foram transformadas nos respectivos escores $z$ segundo sexo e idade. Utilizou-se para análise de médias o teste "t" de Student; ANOVA para análise de variância. O Qui-quadrado foi utilizado no caso das análises das frequências.

O IMC relação de peso em kilogramas divido pelo quadrado da estatura em metros, foi obtido para todas as crianças. 15

A pesquisa foi aprovada pela Comissão de Ética da Faculdade de Saúde Pública da Universidade de São Paulo e obedeceu a todos os preceitos da resolução 196/96 do Conselho Nacional de Saúde em todas as fases de sua execução.

\section{Resultados}

A Tabela 1 apresenta a distribuição amostral em relação ao universo das crianças matriculadas, segundo sexo e evidencia um discreto predomínio do sexo feminino nas faixas etárias maiores sem, contudo, apresentar diferenças estatísticas significativas.

A Tabela 2, que mostra a distribuição do indicador peso para a idade em escores $z$, segundo idade das crianças, evidencia que a média geral e a mediana estão deslocadas para a direita do referencial. Nota-se que apenas duas crianças $(0,58 \%)$ estão entre -2 e $-2,99$ escore $z$ de peso para a idade, e, em 
Tabela 1

Distribuição dos pré-escolares matriculados e selecionados das Escolas Municipais de Educação Infantil (EMEls) segundo sexo e idade em número absoluto e percentual. Mogi-Guaçú, São Paulo.

\begin{tabular}{|c|c|c|c|c|c|c|}
\hline \multirow{4}{*}{ Idade } & \multicolumn{4}{|c|}{ Sexo } & \multirow{3}{*}{\multicolumn{2}{|c|}{ Total }} \\
\hline & \multicolumn{2}{|c|}{ Masculino } & \multicolumn{2}{|c|}{ Feminino } & & \\
\hline & \multicolumn{2}{|c|}{ Matriculadas selecionadas } & \multicolumn{2}{|c|}{ Matriculadas selecionadas } & & \\
\hline & $\mathrm{n}$ & $\%$ & $\mathrm{n}$ & $\%$ & $\mathrm{n}$ & $\%$ \\
\hline$<5$ anos & 26 & 50,00 & 26 & 50,00 & 52 & 100,00 \\
\hline 5 a 5,99 anos & 57 & 47,88 & 70 & 55,12 & 127 & 100,00 \\
\hline$\geq 6$ anos & 83 & 49,40 & 85 & 50,60 & 168 & 100,00 \\
\hline Total & 166 & 47,84 & 181 & 52,16 & 347 & 100,00 \\
\hline
\end{tabular}

Qui-quadrado com dois graus de liberdade masculino: 0,29 $(p>0,50)$ e feminino: 0,28 $(p>0,50)$.

Tabela 2

Distribuição do z-escore de peso para idade segundo idade dos pré-escolares matriculados e selecionados em número absoluto e percentual nas Escolas Municipais de Educação Infantil. Mogi-Guaçú, São Paulo.

\begin{tabular}{|c|c|c|c|c|c|c|c|c|c|c|c|c|c|c|c|c|c|c|}
\hline \multirow[t]{3}{*}{ Idade } & \multicolumn{16}{|c|}{ Escore z - Peso/Idade } & \multirow{2}{*}{\multicolumn{2}{|c|}{ Total }} \\
\hline & \multicolumn{2}{|c|}{$\leq 3,00$} & \multicolumn{2}{|c|}{$-2,99$ a -2} & \multicolumn{2}{|c|}{$-1,99$ a -1} & \multicolumn{2}{|c|}{$-0,99$ a 0} & \multicolumn{2}{|c|}{0,01 a 1} & \multicolumn{2}{|c|}{1,01 a 2} & \multicolumn{2}{|c|}{2,01 a 3} & \multicolumn{2}{|c|}{$>3,00$} & & \\
\hline & $n$ & $\%$ & $n$ & $\%$ & $n$ & $\%$ & $n$ & $\%$ & $n$ & $\%$ & $n$ & $\%$ & $n$ & $\%$ & $n$ & $\%$ & $n$ & $\%$ \\
\hline$<5$ anos & - & - & 1 & 1,92 & 2 & 3,85 & 12 & 23,08 & 17 & 32,69 & 13 & 25,00 & 3 & 5,77 & 4 & 7,69 & 52 & 100,00 \\
\hline 5 a 5,99 anos & - & - & 1 & 0,79 & 15 & 11,81 & 43 & 33,86 & 41 & 32,28 & 18 & 14,17 & 4 & 3,15 & 5 & 3,94 & 127 & 100,00 \\
\hline$\geq 6$ anos & - & - & - & - & 16 & 9,52 & 37 & 22,02 & 61 & 36,32 & 35 & 20,83 & 8 & 4,76 & 11 & 6,55 & 168 & 100,00 \\
\hline Total & - & - & 2 & 0,58 & 33 & 9,51 & 92 & 26,51 & 119 & 34,30 & 66 & 19,02 & 15 & 4,32 & 20 & 5,76 & 347 & 100,00 \\
\hline
\end{tabular}

Qui-quadrado: 14,46 (12 graus de liberdade); $p=0,272$

Tabela 3

Distribuição do z-escore de altura para idade, segundo idade dos pré-escolares matriculados e selecionados das Escolas Municipais de Educação Infantil em número absoluto e percentual. Mogi-Guaçú, São Paulo.

\begin{tabular}{|c|c|c|c|c|c|c|c|c|c|c|c|c|c|c|c|c|c|c|}
\hline \multirow[t]{3}{*}{ Idade } & \multicolumn{16}{|c|}{ Escore z - Altura/Idade } & \multicolumn{2}{|r|}{ Total } \\
\hline & \multicolumn{2}{|c|}{$\leq 3,00$} & \multicolumn{2}{|c|}{$-2,99$ a -2} & \multicolumn{2}{|c|}{$-1,99 a-1$} & \multicolumn{2}{|c|}{$-0,99$ a 0} & \multicolumn{2}{|c|}{0,01 a 1} & \multicolumn{2}{|c|}{1,01 a 2} & \multicolumn{2}{|c|}{2,01 a 3} & \multicolumn{2}{|c|}{$>3,00$} & & \\
\hline & $\mathrm{n}$ & $\%$ & $n$ & $\%$ & $\mathrm{n}$ & $\%$ & $n$ & $\%$ & $n$ & $\%$ & $n$ & $\%$ & $\mathrm{n}$ & $\%$ & $\mathrm{n}$ & $\%$ & $n$ & $\%$ \\
\hline$<5$ anos & - & - & - & - & 5 & 9,61 & 11 & 21,16 & 23 & 44,23 & 10 & 19,23 & 2 & 3,85 & 1 & 1,92 & 52 & 100,00 \\
\hline 5 a 5,99 anos & - & - & 2 & 1,57 & 20 & 15,75 & 39 & 30,71 & 37 & 29,13 & 24 & 18,90 & 4 & 3,15 & 1 & 0,79 & 127 & 100,00 \\
\hline$\geq 6$ anos & - & - & 2 & 1,19 & 8 & 4,76 & 62 & 36,91 & 61 & 36,31 & 29 & 17,26 & 6 & 3,57 & - & - & 168 & 100,00 \\
\hline Total & - & - & 4 & 1,15 & 33 & 9,51 & 112 & 32,28 & 121 & 34,86 & 63 & 18,16 & 12 & 3,46 & 2 & 0,58 & 347 & 100,00 \\
\hline
\end{tabular}

Qui-quadrado: 18,70 (12 graus de liberdade); $p=0,096$ 
Tabela 4

Distribuição dos pré-escolares de acordo com os pontos de corte do índice de massa corporal (IMC) proposto por Cole et al. para o sobrepeso e obesidade das crianças segundo idade em anos.

\begin{tabular}{|c|c|c|c|c|c|c|c|c|}
\hline \multirow{3}{*}{ Idade } & \multicolumn{6}{|c|}{ Índice de Massa Corporal } & & \\
\hline & \multicolumn{2}{|c|}{ Normal } & \multicolumn{2}{|c|}{ Sobrepeso } & \multicolumn{2}{|c|}{ Obesidade } & \multicolumn{2}{|c|}{ Total } \\
\hline & $\mathrm{n}$ & $\%$ & $\mathrm{n}$ & $\%$ & $\mathrm{n}$ & $\%$ & $\mathrm{~N}$ & $\%$ \\
\hline$<5$ anos & 33 & 63,46 & 12 & 23,08 & 7 & 13,46 & 52 & 100,00 \\
\hline 5 a 5,99 anos & 107 & 84,25 & 16 & 12,60 & 4 & 3,15 & 127 & 100,00 \\
\hline$\geq 6$ anos & 116 & 69,05 & 37 & 22,02 & 15 & 8,93 & 168 & 100,00 \\
\hline Total & 256 & 73,78 & 65 & 18,73 & 26 & 7,49 & 347 & 100,00 \\
\hline
\end{tabular}

Fonte: Cole et al. Establishing a standart definition. BMJ. 2000.15

\section{Figura 1}

Curvas de distrubuição percentual de sobrepeso e obesidade das crianças conforme o escore $z$. Mogi-Guaçu, São Paulo.

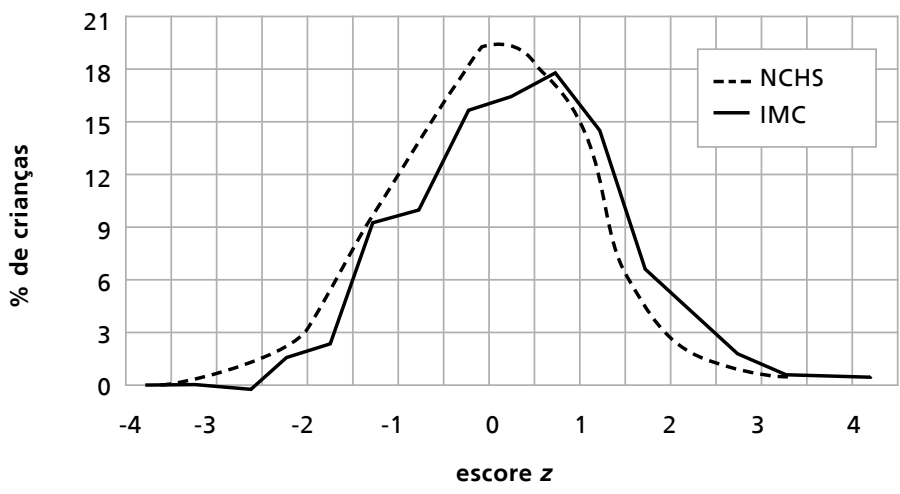

NCHS = National Center for Health Statistics; IMC = índice de massa corporal

contrapartida, 35 crianças encontram-se acima do segundo escore $z$ (positivo). Não foi possível identificar uma faixa etária com maior risco de sobrepeso ou obesidade $(p=0,272)$.

Na Tabela 3 percebe-se que a média geral e a mediana do indicador altura para a idade, encontram-se entre 0,01 a 1 escore $z$, sugerindo, novamente, a tendência de deslocamento da curva para a direita. O indicador não mostra associação significativa com a idade das crianças matriculadas $(p=0,096)$.

A Tabela 4 nos mostra que, do total das crianças pesquisadas, aproximadamente $1 / 4(91 / 347)$ encontrase acima do peso adequado para idade, quando se leva em consideração o IMC. Dessas crianças, em torno de $71 \%$ (65/91) estavam igual ou além do percentil 85, e 29\% (29/91) do percentil 95.
A Figura 1 mostra a tendência de sobrepeso e obesidade dos pré-escolares, expresso no deslocamento dos valores do IMC para a direita da mediana do referencial National Center for Health Statistics (NCHS).

\section{Discussão}

Várias pesquisas enfatizam o aumento de peso de crianças nos últimos anos,16-18. No Brasil, a rápida diminuição das taxas de desnutrição associada ao aumento nas taxas de obesidade tem ocorrido em curto intervalo de tempo, agregando uma nova preocupação, no âmbito das políticas públicas, que envolve os cuidados alimentares e nutricionais com as crianças. É preciso destacar que o maior risco, em longo prazo, da obesidade infantil, é sua persistência no adulto, com todas as consequiências associadas para a saúde. ${ }^{19} \mathrm{O}$ crescente aumento do número de indivíduos obesos parece estar mais relacionado às mudanças no estilo de vida e aos hábitos alimentares. 20,21 Torriente et al. ${ }^{22}$ referem que nos últimos anos há uma epidemia de inatividade, explicando o aumento da prevalência da obesidade.

Do ponto de vista biológico, a obesidade infantil vem sendo apontada como fator de risco à doenças do aparelho circulatório, ortopédico e metabólicas. ${ }^{23-25}$ Segundo Machado et al. ${ }^{26}$ a preocupação com a obesidade das crianças deve ser também levada ao campo das performances motoras ao longo do seu desenvolvimento psicossocial. Ainda neste aspecto, Azevedo 27 já alertava sobre a necessidade de estudos sobre os aspectos psíquicos da criança obesa.

No tocante a susceptibilidade biológica individual, dentre os fatores predisponentes ao sobre- 
peso e obesidade cita-se: balanço energético positivo, fatores relativos à dieta, influência sócio-ambiental e hereditariedade.

No caso específico desta pesquisa, é pouco provável que a situação nutricional verificada tenha ocorrido em função de mudanças genéticas da população. Embora exista predisposição genética à obesidade (possivelmente através de carga autossômicorecessiva) Takashi, ${ }^{5}$ Habicht et al., 28 e Jeff 29 a relativizaram na determinação do estado nutricional das crianças em face de forte influência do ambiente no fenótipo humano. Nesse sentido, cabe lembrar que em Mogi-Guaçú as condições mínimas de saúde estão equacionadas: a rede de água encanada atinge 99\% de cobertura; o acesso à rede de Saúde tem grande cobertura populacional e não há moradores em favelas ou cortiços. ${ }^{3}$ Essas informações se coadunam com as expectativas de Monteiro e Conde 9 sobre os fatores explicativos da transição nutricional das crianças de São Paulo.

A utilização do indicador peso/idade é preconizada pelo Ministério da Saúde do Brasil para avaliar o risco de sobrepeso e obesidade no âmbito individual. Contudo, as recomendações recentes do Center for Disease Control (CDC), de Atlanta, Estados Unidos da América do Norte, 15 apontam o índice de massa corporal como método para avaliar a situação de sobrepeso ou obesidade em crianças, a partir de dois anos de idade. Tal indicador teria a vantagem de incorporar o valor da estatura da criança na caracterização do estado nutricional. A disponibilização pelo National Center for Health Statistics, de um referencial para o IMC em crianças serve de impulso para o diagnóstico precoce da obesidade, seja em âmbito individual, seja coletivo, colocando assim o tratamento desse agravo na agenda das ações de saúde pública.

\section{Referências}

1. Mason JB, Habicht JP, Tabatabai H, Valverde V. Nutritional surveillance. Geneva: World Health Organization; 1984.

2. Tanner JM. A concise history of growth studies from Buffon to Boas. In: Falkner F, Tanner JM. Human growth. New York: Plenum; 1978. v. 3, p. 515-93.

3. Jelliffe DB. Evaluación del estado de nutrición de la comunidad. Ginebra: Organización Mundial de la Salud; 1968.

4. Owen GM. The assessment and recording of measurements of growth of children; report of small conference. Pediatrics. 1973; 51: 461-5.

5. Takahashi E. Growth and environmental factors in Japan. Hum Biol. 1966; 38: 112-30.

6. Nóbrega FJ. Influência do peso ao nascimento e de variáveis maternas na determinação da estatura em crianças. J Pediatria. [Rio J] 1991; 67: 163-7.
A pesquisa constatou que no escore $z$ do indicador peso/idade houve uma concentração das crianças para o lado direito da curva normal em comparação à referência. Isso indica uma tendência à obesidade entre as crianças avaliadas. Silva et al.,30 estudando a demanda de crianças atendidas em ambulatório, concluíram que as prevalências de sobrepeso e obesidade foram elevadas entre préescolares. O citado estudo, com validade externa para famílias de classe socioeconômica baixa, reforça os resultados encontrados em Mogi-Guaçú, de abrangência socioeconômica maior. Os achados endossam a idéia de que a obesidade infantil vem se tornando um problema de saúde pública.

Um dos mais importantes parâmetros de qualidade de vida da população é o crescimento em altura de crianças.31,32 No presente estudo encontramos dados, que se coadunam com outros trabalhos como o de Monteiro e Conde, 10 nos quais há uma tendência de deslocamento da curva para a direita dos indicadores antropométricos, como é o caso do indicador estatura para a idade em crianças.

Finalmente, os achados neste estudo sugerem que há, em Mogi-Guaçú, uma nova agenda nutricional no tocante às prioridades em saúde pública. Por um lado, o sobrepeso e obesidade surgem como elementos de reflexão na prática diária dos nutricionistas responsáveis pelas dietas das pré-escolas e também como preocupação entre os demais profissionais de saúde das equipes responsáveis pelo atendimento direto das crianças e/ou das famílias; por outro lado, a diminuição das taxas de desnutrição deslocam a atenção a este problema no sentido da busca ativa de pacientes, bem como, na organização e planejamento de programas individualizados de recuperação nutricional.
7. Galuska DA, Serdula M, Pamuk E, Siegel PZ, Byers T. Trends in overweight among US adults from 1987 to 1993: a multistate telephone survey. Am J Public Health. 1996; 86: $1729-35$.

8. Bundred P, Kitchiner D, Buchan I. Prevalence of overweight and obese children between 1989 and 1998: population based series of cross sectional studies. BMJ. 2001; 322: $1-4$.

9. Monteiro CA, Conde WL. Tendência secular da desnutrição e da obesidade na infância na cidade de São Paulo (19741996). Rev Saúde Pública. 2000; 34 (Supl 6): 52-61.

10. Monteiro CA, Conde WL. Tendência secular do crescimento pós-natal na cidade de São Paulo (1974-1996). Rev Saúde Pública. 2000; 34 (Supl 6): 41-51. 
11. Khan AA, Gupta BM. Social and economic factors in malnourished children around Lazaka, Zambia. Trop Geogr Med. 1977; 29: 283-7.

12. Ogden CL, Flegal KM, Carroll MD, Johnson CL. Prevalence and trends in overweight among US children and adolescentes, 1999-2000. JAMA. 2002; 288: 1772-3.

13. Mogi-Guaçú. São Paulo. Secretaria da Comunicação Social. Dados sobre o município. Mogi-Guaçú; 2002.

14. OMS (Organización Mundial de la Salud). El estado físico: uso e interpretación de la antropometría: informe de un Comité de Expertos de la OMS. Ginebra; 1995. (Serie de Informes Técnicos, 854).

15. Cole TJ, Bellizzi MC, Flegal KM, Dietz WH. Establishing a standard definition for child overweight and obesity worldwide: international survey. BMJ. 2000; 320: 1-6.

16. Tremblay MS, Willms JD. Secular trends in the body mass index of Canadian children. CMAJ. 2000; 163: 1429-33.

17. Kain J, Uauy R, Vio F, Albala C. Trends in overweight and obesity prevalence in Chilean children: comparison of three definitions. Eur J Clin Nutr. 2002; 56: 200-4.

18. Herpertz-Dahlmann B, Geller F, Bohle C, Khalil C, TrostBrinkhues GZA, Hebebrand J. Secular trends in body mass index measurements in preschool children from the City of Aachen, Germany. Eur J Pediatr. 2003; 162: 104-9.

19. Guo SS, Roche AF, Chumlea WC, Gardner JD, Siervogel RM. The predictive value of childhood body mass index values for overweight at age 35 years. Am J Clin Nutr. 1994; 59: 810-9.

20. Rosenbaum M, Leibel RL. The physiology of body weight regulation: relevance to the etiology of obesity in children. Pediatrics. 1998; 101: 525-39.

21. Dennison BA, Erb TA, Jenkins PL. Television viewing and television in bedroom associated with overweight risk among low-income preschool children. Pediatrics. 2002; 109: 1028-35.

22. Torriente GMZ, Molina DC, Díaz Y, Fernandez AT, Argüelles XH. Obesidad en la infancia: diagnóstico y tratamiento. Rev Cubana Pediatr. 2002; 74: 233-9.

23. Azcona C, Patino A, Ramos M, Ruza E, Raggio S, Alonso L. Obesidad infantil. Rev Med Univ Navarra. 2000; 44: 2944.

Recebido em 7 de dezembro de 2004

Versão final apresentada em 15 de março de 2006

Aprovado em 2 de maio de 2006
24. Burrows R, Gattas V, Leiva L, Barrera G, Burgueno M. Biological, familial and metabolic characteristics of infantile and juvenile obesity. Rev Med Chil. 2001; 129: 115562.

25. Moser M. Obesity and hypertension in children; caffeine, stress, and elevated blood pressure; resistant hypertension; and is it lowering of blood pressure alone that account for reduction in cardiovascular events? J Clin Hypertens. (Greenwich) 2001; 3: 343-5.

26. Machado HS, Campos W, Silva SG. Relação entre composição corporal e a performance de padrões motores fundamentais em escolares. Rev Bras Ativ Fis Saúde. 2002; 7: $63-70$.

27. Azevedo MASB. Um estudo exploratório da personalidade da criança obesa através do desenho da figura humana e dos indicadores emocionais de Koppitz [tese doutorado]. Campinas: Faculdade de Ciências Médicas da Universidade Estadual de Campinas; 1997.

28. Habicht JP, Martorell R, Yarbrough C, Malina RM, Klein RE. Height and weight standards for preschool children. How relevant are ethnic differences in growth potential? Lancet. 1974; 1: 611-4.

29. Jeff S. Marriage, obesity and dieting. In: Kallen D, Sussman M. Obesity and the family. New York: Haworth; 1984. p. 115-39.

30. Silva GAP, Balaban G, Baracho JDS, Freitas MMV, Nascimento EMM. Prevalência de sobrepeso e obesidade entre pré-escolares atendidos no ambulatório do Hospital das Clínicas/UFPE - Recife, Pernambuco - Brasil. An Fac Med Univ Fed Pernambuco. 2001; 46: 127-31.

31. Gopalan C. Stunting: significance and implications for public health policy. In: Waterlow JC. Linear growth retardation in less developed countries. New York: Raven Press; 1988. p. 265-84. (Nestlé Nutrition Workshop Series, 14).

32. Martorell R, Mendoza F, Castillo R. Poverty and stature in children. In: Waterlow JC. Linear growth retardation in less developed countries. New York: Raven Press; 1986. p. $57-$ 73. (Nestlé Nutrition Workshop Series, 14). 